\title{
EEG spectral features discriminate between Alzheimer's and vascular dementia
}

\section{Emanuel Neto ${ }^{1,2}$, Elena A. Allen ${ }^{1,3,4}$, Harald Aurlien ${ }^{2}$, Helge Nordby ${ }^{1}$ and Tom Eichele ${ }^{1,2,3}$}

${ }^{1}$ Institute of Biological and Medical Psychology, University of Bergen, Bergen, Norway

2 Section for Clinical Neurophysiology, Haukeland University Hospital, Bergen, Norway

${ }^{3}$ K. G. Jebsen Center for Research on Neuropsychiatric Disorders, Bergen, Norway

${ }^{4}$ The Mind Research Network, Albuquerque, NM, USA

\section{Edited by:}

Srikantan S. Nagarajan, University of California San Francisco, USA

Reviewed by:

Anand Joshi, University of Southern California, USA

Alberto Sorrentino, Universita di

Genova, Italy

Kamalini Gayathree Ranasinghe,

University of California San Francisco, USA

${ }^{*}$ Correspondence:

Emanuel Neto, Institute of Biological and Medical Psychology, University of

Bergen, Bergen, Norway

e-mail: netoemanuel@gmail.com
Alzheimer's disease (AD) and vascular dementia $(\mathrm{VaD})$ present with similar clinical symptoms of cognitive decline, but the underlying pathophysiological mechanisms differ. To determine whether clinical electroencephalography (EEG) can provide information relevant to discriminate between these diagnoses, we used quantitative EEG analysis to compare the spectra between non-medicated patients with $\mathrm{AD}(n=77)$ and $\mathrm{VaD}(n=77)$ and healthy elderly normal controls (NC) $(n=77)$. We use curve-fitting with a combination of a power loss and Gaussian function to model the averaged resting-state spectra of each EEG channel extracting six parameters. We assessed the performance of our model and tested the extracted parameters for group differentiation. We performed regression analysis in a multivariate analysis of covariance with group, age, gender, and number of epochs as predictors and further explored the topographical group differences with pair-wise contrasts. Significant topographical differences between the groups were found in several of the extracted features. Both $A D$ and $\mathrm{VaD}$ groups showed increased delta power when compared to NC, whereas the AD patients showed a decrease in alpha power for occipital and temporal regions when compared with NC. The VaD patients had higher alpha power than $N C$ and $A D$. The $A D$ and $V a D$ groups showed slowing of the alpha rhythm. Variability of the alpha frequency was wider for both $A D$ and $V a D$ groups. There was a general decrease in beta power for both $\mathrm{AD}$ and $\mathrm{VaD}$. The proposed model is useful to parameterize spectra, which allowed extracting relevant clinical EEG key features that move toward simple and interpretable diagnostic criteria.

Keywords: Alzheimer's disease, vascular dementia, electroencephalogram, qEEG, quantitative analysis

\section{INTRODUCTION}

Alzheimer's disease $(\mathrm{AD})$ is a debilitating neuro-degenerative disease, and is one of the most common forms of dementia among the elderly population (1) with a significant socio-economic burden for societies in developed countries. AD particularly affects individuals over the age of 65 years and it is estimated that its prevalence will triple within the next 40 years $(2,3)$. Vascular dementia $(\mathrm{VaD})$ may result either from ischemic or hemorrhagic cerebrovascular disease (CVD), or from cardiovascular or circulatory disturbances that injure brain regions relevant to memory, cognition, and behavior (4). $\mathrm{VaD}$ is the second most common form of dementia after $\mathrm{AD}$, affecting approximately $20 \%$ of the dementia cases worldwide (5). While VaD can be assessed with the use of imaging techniques at early stages of the disease, the similarities between symptoms between the different conditions can lead to diagnostic uncertainty. Autopsy assessment studies in dementia report that $\mathrm{VaD}$ was present in $24-28 \%$ of $\mathrm{AD}$ cases $(6,7)$. One current difficulty is the relative lack of specific biomarkers; certain diagnosis for $\mathrm{AD}$ is still only possible through a post-mortem exam; routinely several examinations, such as the mini-mental state examination (MMSE), positron emission tomography (PET), computer tomography (CT), magnetic resonance imaging (MRI), and electroencephalogram (EEG) are used during differential diagnostics $(8,9)$. So far, clinical EEG assessment is mainly performed through visual inspection, identifying clear signs of pathology, while usually not considering other quantifiable measures. Potentially relevant features that are not immediately visible, such as power modulations, connectivity changes, or sparse small amplitude phenomena, may thus be overlooked.

Quantitative EEG analysis therefore may be helpful in the clinical context. For example, it is well known that decreases of alpha and beta power and increases of the delta and theta frequencies are related to brain pathology and general cognitive decline (10-13). Recent studies have demonstrated that AD has a pre-symptomatic phase that can last for years, known as mild cognitive impairment (MCI) and while neuronal degeneration is taking place, the clinical symptoms remain subtle. Consequently, early behavioral and pharmacological interventions, which can ameliorate the course of the disease, should not be administered based on clinical data alone (14-18). However, abundant literature reports that specific drugs induce alterations on electroencephalographic readings. A comprehensive overview of recent studies clusters typical effects with different pharmacological agents (19). Specifically, drugs that act 
on the nervous system such as psycholeptics and psychoanaleptics may induce neuronal hyperexcitability or drowsiness and hence EEG patterns change (20). Nevertheless, it has been shown that the resting EEG activity can predict future cognitive decline or conversion to dementia in MCI subjects with high accuracy (21-25). Furthermore, recent studies suggest that spectral analysis can be used to distinguish AD from other dementias (26-29). These studies use various EEG markers such as spectral power, coherence, and frequency of rhythms in delta, theta, alpha, or beta bands, which are considered valuable markers for group classification by several studies (11, 30-35). However, the more EEG features studied, the larger sample sizes are required, which is often not easy to obtain. Moreover, many EEG studies using qEEG analysis for classification of AD differ on the test-paradigm, sample size, methods, features extracted, and classification models (36). The implementation of systematic guidelines to access dementia through the use of core EEG markers would facilitate these methods and provide more material for research.

In an effort to meet the needs of clinical EEG community, this study proposes a model for extracting EEG spectral features in groups of dementia patients, which might also be used as biomarkers for other diseases involving encephalopathy. This study shows the potential to differentiate between two of the most common types of dementias $(\mathrm{AD}$ and $\mathrm{VaD})$ at the group level. In particular, we performed quantitative spectral analysis on clinical EEGs collected from a large in-house database (37), which holds over 30,000 EEG records. We applied a curve-fitting algorithm to model the frequency spectrum of each patient, extracting a total of six parameters from each channel. These features represent low (delta) and high (beta) frequency bands, decay of amplitude from low to higher frequencies, alpha power, alpha frequency, and dispersion of alpha.

\section{MATERIALS AND METHODS \\ DESIGN}

We performed a retrospective analysis of $\mathrm{AD}$ and $\mathrm{VaD}$ patients, examining EEGs that were collected as part of clinical diagnostic procedure.

\section{SAMPLE}

The database of the neurophysiology department of Haukeland University Hospital contains more than 37,000 EEG datasets from about 23,000 subjects available internally for research. From this database, we initially selected a convenience sample of all datasets from outpatients diagnosed with $\mathrm{AD}(n=534)$, with international classification disease (ICD) codes ICD-10, F00.x and G30.x; $\operatorname{VaD}(n=203)$ (ICD-10 F01.X), and NC $(n=3138)$ of nonhospitalized individuals at the time of the EEG recording, free of medication, and with no brain disease on record. The mean age and SD of the initial sample were $74.34 \pm 9.92,72.41 \pm 11.16$, and $52.2 \pm 14.38$ years for $\mathrm{AD}, \mathrm{VaD}$, and $\mathrm{NC}$, respectively. We applied stringent criteria for inclusion and exclusion of samples as described in Sections "Selection Criteria" and "Pre-processing," remaining with a total of 242,88 , and 1950 datasets on $\mathrm{AD}, \mathrm{VaD}$, and NC groups, respectively. The samples were then age and gender matched, leaving 77 cases in each group, with an average age of $73.1 \pm 10.4$ years and $51 \%$ males.

\section{Selection criteria}

For the initial sample described above, we applied the same inclusion criteria to all groups. First, we excluded patients with ages below 35 years old at the time of the recording. Second, we excluded the EEG recordings performed when subjects were hospitalized. Lastly, we excluded multiple EEG datasets from each patient including only the latest EEG dataset from each.

A general exclusion rule was applied when incongruent, insufficient, or poor data quality was found on the patient's clinical or personal information, which excluded 988 patients. We considered the medications prescribed to each patient at the time of EEG recording, and excluded patients who were taking anticholinergic and dopaminergic agents, antipsychotics, anxiolytics, hypnotics and sedatives, anti-depressants, and psychostimulants. A complete list of the medications prescribed was resumed in Table S1 in Supplementary Material. The entire clinical diagnose history available from each patient's record was taken into consideration: for the patients belonging to NC, we excluded those who had been diagnosed at any time point (prior or post the EEG recording date) with any CVD, any mental, behavioral, or neurodevelopmental disorders. For the AD and VaD groups, we excluded those who had been diagnosed at any time with any other brain or CNS-related disorder. In particular, the $\mathrm{AD}$ and $\mathrm{VaD}$ groups contained datasets from patients who had been only diagnosed with Alzheimer disease or $\mathrm{VaD}$, respectively, before or persistently after the EEG recording date. For further details on the selection criteria, please see Table S2 in Supplementary Material. From the initial sample, we considered 2092, 269, and 118 datasets for NC, AD, and VaD groups, respectively, which were submitted to the pre-processing. A total of 199 datasets were excluded during the pre-processing due to insufficient epochs (see next section). The final sample was constituted by 77 age- and gender-matched subjects on each group with age averages of $71.5 \pm 11.2,73.5 \pm 9.9$, and $74.1 \pm 9.9$, respectively. The gender distribution was 49,51 , and $52 \%$ males for $\mathrm{NC}, \mathrm{AD}$, and $\mathrm{VaD}$ groups, respectively.

\section{Pre-processing}

All EEG datasets were acquired using 22 channels positioned in 10-20 system placements (Fp1, Fpz, Fp2, F7, F3, Fz, F4, F8, T3/T7, C3, Cz, C4, T4/T8, T5/P7, P3, Pz, P4, T6/P8, O1, O2, M1, M2) acquired at $128 \mathrm{~Hz}(n=59), 256 \mathrm{~Hz}(n=164)$, and $500 \mathrm{~Hz}(n=8)$ using NicoletOne ${ }^{\mathrm{TM}} \mathrm{EEG}$ system. Input impedances were set to $Z>100 \mathrm{M} \Omega$. Hardware single pole high-pass $(0.16 \mathrm{~Hz} \pm 10 \%)$ and low-pass $(500 \mathrm{~Hz} \pm 10 \%)$ filters were applied individually to each channel before pre-amplification. EEGs were stored under raw format in the database. All the pre-processing and data analysis were performed in the Mathworks ${ }^{\circledR}$ Matlab environment. EEG raw files were imported to the EEGLab v.10.1.1.0b toolbox (38) using an in-house data-reader. Data were resampled to $256 \mathrm{~Hz}$. From the standard clinical EEG recording protocol that lasts for $20 \mathrm{~min}$ and includes eye open/closed conditions, hyperventilation, and provocations with photic stimulation, we restricted the input data for analysis to the first $9 \mathrm{~min}$, which contained only the alternating eyes open/closed resting conditions. A 1536-points high-pass-band filter was applied with cut-off frequency of 0.5 and a low-pass filter with cut-off of $50 \mathrm{~Hz}$ using a standard least square linear-phase FIR filter design. EEGs were segmented into non-overlapping epochs 
of $1 \mathrm{~s}$ that were evaluated for possible rejection using automatic amplitude, power, and statistical thresholding. The remaining segments were subjected to an individual independent component analysis (ICA) using the Infomax algorithm with 15 components in order to identify and remove residual contributions from eye movements. Four spatial templates for these types of artifacts were generated using 24 randomly drawn datasets, in which the corresponding artifact maps were visually identified and averaged across subjects to form the templates. Figure 1 displays the eye movement templates. The ICA topographies from each EEG dataset were correlated against the artifact templates and were removed if an absolute correlation coefficient above 0.8 was found. The continuous data were reconstructed from the non-artifact components and then segmented into $2 \mathrm{~s}$ epochs with $1 \mathrm{~s}$ overlap, which is equivalent to the Welch's procedure (39) with a rectangular windows and 50\% segment overlap. Subsequently, the data were transformed into the frequency domain using fast Fourier transform (FFT). Since the frequency spectrum selected for the pre-processing was from 0.5 to $50 \mathrm{~Hz}$, we obtained 100 frequency data points for the 22 channels and a variable number of epochs for each dataset subjected for analysis. The spatial standard deviation (sSTD) index of each epoch was calculated across the 22 channels in the frequency domain according to (40) and $z$-scored. Epochs where the sSTD index exceeded $|z|>1$ were excluded from further analysis, as illustrated in Figure 2. With the exclusion of such epochs, we ended up with a limited number of available segments of data for the initial sample. In order to unify the amount of data across subjects for the final sample, we determined the minimum common number of existing epochs across subjects that maximized the inclusion. This resulted in the selection of 334 epochs of data from each dataset (approx. $5 \mathrm{~m} 30 \mathrm{~s}$ ). Subjects with fewer than 334 epochs were excluded from the initial sample.

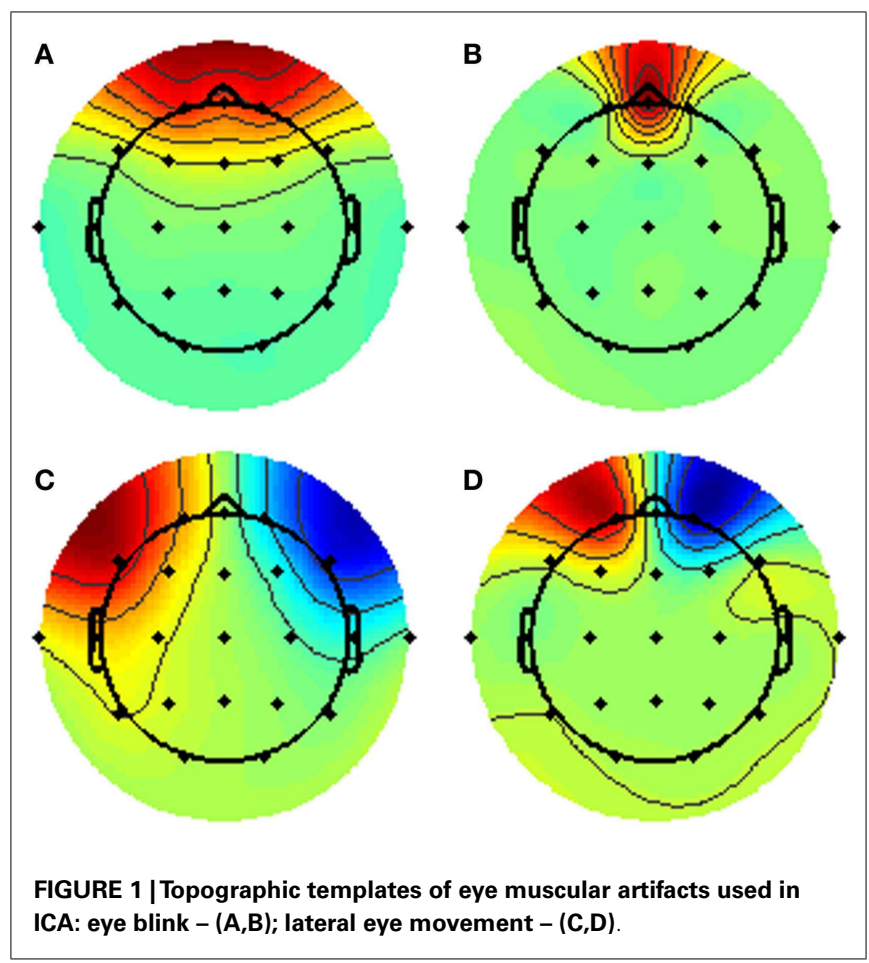

The available epochs of the final sample followed a normal distribution across the three groups with averages of $386 \pm 24$ for the $\mathrm{NC}$ group, $391 \pm 28$ epochs for $\mathrm{AD}$, and $387 \pm 20$ for the $\mathrm{VaD}$. No significant differences were found in the amount of available segments of data between the three groups $(F=1.03, p=0.43)$. Epochs that contained higher amplitude in the range of the alpha band $(8-12 \mathrm{~Hz})$, were given priority for inclusion and remaining epochs were discarded. In this way, we expected to maximize the expression of the alpha frequency in the analysis. After age-gender matching of the groups, the final sample used in the analysis was composed of 334 epochs of data for each subject and 77 subjects in each group.

\section{GROUP SPECTRUM DIFFERENCES}

To display the general differences between groups, we averaged the spectra first over all epochs within subjects, then over all subjects within each group. The mean spectrum \pm 1 standard error of the mean (SEM) for each group are displayed in Figure 3. Further spectrum differences were explored using a curve-fitting model for comparison

\section{CURVE FITTING}

To describe the power spectrum in a concise way, we combined a power-loss function modeling the roughly $1 / f$ drop-off and a Gaussian function centered on the alpha-frequency peak. Specifically, we modeled the spectral power, $P(f)$, with the following expression:

$$
P(f)=S f^{-k}+A e^{-\frac{(f-c)^{2}}{w}}+b
$$

All parameters were estimated using a non-linear least squares trust-region optimization algorithm, as provided in Matlab's fitcurve toolbox. The model was fit to the power spectrum (averaged across epochs) in the frequency range of $1-30 \mathrm{~Hz}$, to avoid tapering effects caused by the low-pass filter $(0.5 \mathrm{~Hz})$. We applied a particular exclusion rule for the fit of the power-loss function,

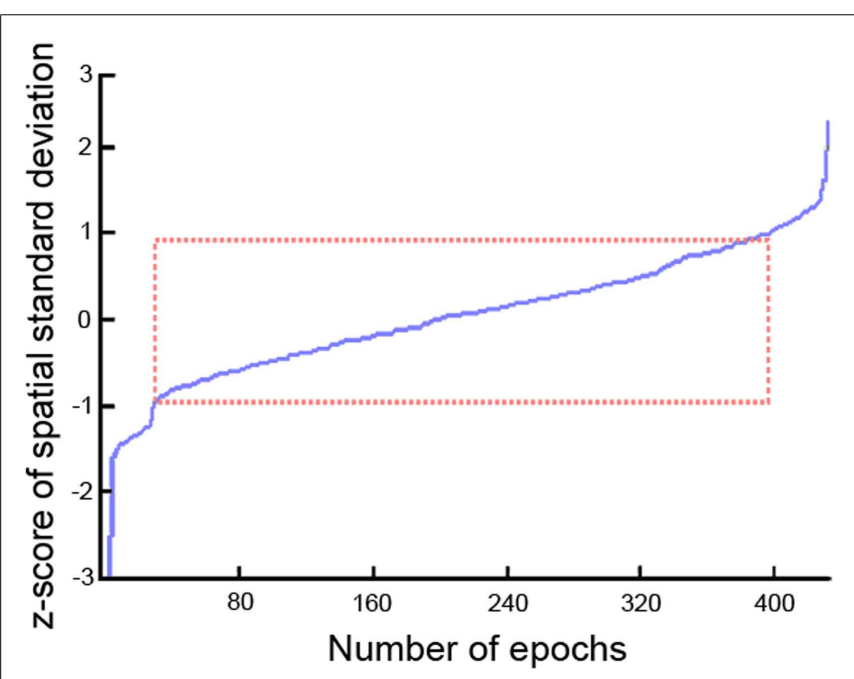

FIGURE 2 | Discrimination of epochs using the spatial standard deviation (sSTD) index. 


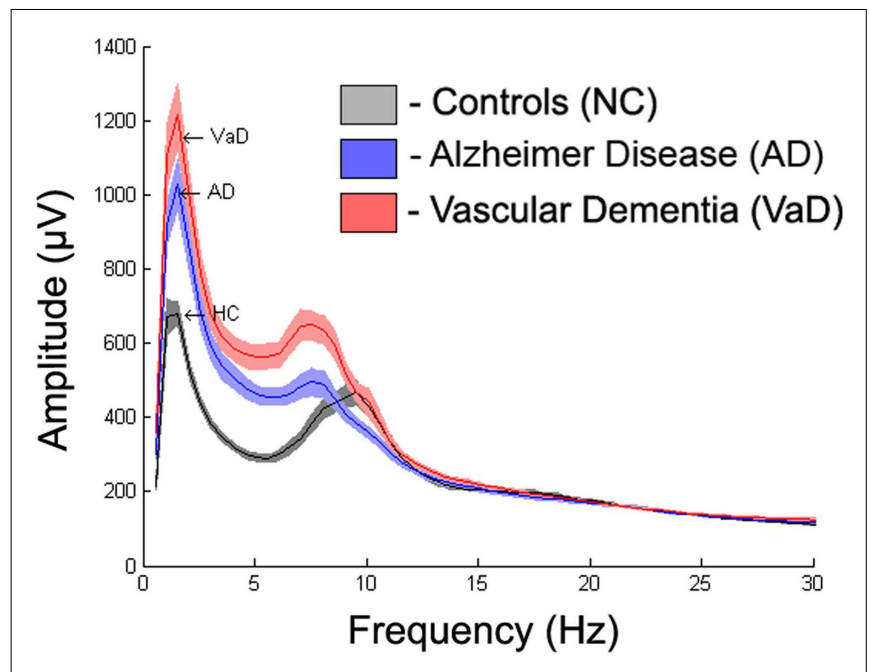

FIGURE 3 | Averaged frequency spectrum over all channels for each group with absolute frequency.

excluding data points that correspond to the alpha band range (7$13 \mathrm{~Hz}$ ). For the fitting curve algorithm, upper and lower estimation boundaries for each of the parameters were determined based on fits to the grand average spectra and are provided in Table 1. The goodness-of-fit of the model was assessed with the $R^{2}$ statistic (Figure 4), and examples of best and worst fits are shown in Figure 5. The six parameters used in our model can be related to classic electrophysiological markers. Free parameters $S$ and $k$ relate to the power-loss function and represent its scale and loss, respectively. Values of $S$ represent the amplitude at lower frequencies (delta waves), while $k$ indicates the roughly $1 / f$ decay of amplitude from lower to higher frequencies. Larger values of $k$ denote a faster drop-off in power. Parameters $A, c$, and $w$ relate to the Gaussian and represent the amplitude, center, and dispersion of the alpha peak, respectively. The alpha peak, here characterized by the amplitude, $A$ and the center, $c$, which is often described in the literature as the occipital dominant rhythm (41-44) and is related to brain pathology and may potentially be used as reliable markers for a group differentiation $(45,46)$. Parameter $w$, which represents the dispersion of the alpha peak, has been described previously as the dominant frequency variability (47). Parameter $b$ represents a global offset or baseline power of the entire frequency spectrum where the drop function and Gaussian best fit on and is related with the limit of the high frequency (beta) amplitude. For a general group comparison, we used the results from parameter $c$ to estimate the averaged value of the alpha frequency for each group across all channels.

\section{STATISTICAL ANALYSIS}

Because of the mild covariance between some spectral parameters (see Results), we used a multivariate analysis of covariance (MANCOVA) model as implemented in the MANCOVAN toolbox (42) to understand group differences while controlling for other nuisance factors. Prior to modeling, we used Box-Cox power transforms to improve normality of parameters $S$ and $A$, which were highly skewed. Lambda values (Box-Cox parameters)
Table 1 | Fitting curve algorithm setup with initial, upper and lower boundaries for each of the six parameters.

\begin{tabular}{llccc}
\hline Parameter & Interpretation & Initial & $\begin{array}{l}\text { Lower } \\
\text { limit }\end{array}$ & $\begin{array}{l}\text { Upper } \\
\text { limit }\end{array}$ \\
\hline$S$ & Low frequency power & 1000 & 0 & $\infty$ \\
$k$ & $\begin{array}{l}\text { Decay from lower to } \\
\text { higher frequencies }\end{array}$ & 1 & 0 & 5 \\
Alpha power & 50 & 0 & $\infty$ \\
$C$ & Alpha frequency & 8 & 6 & 14 \\
$W$ & Alpha dispersion & 1 & 0.25 & 25 \\
$b$ & Baseline of the entire & 60 & 0 & $\infty$ \\
& frequency spectrum & & & \\
\hline
\end{tabular}

were determined by maximizing the log-likelihood of normality and were 0.07 and 0.27 , respectively. We first applied a MANCOVA to the spectral parameters averaged over all channels, and used a stepwise regression procedure with backward elimination to remove predictors that did not account for significant variance. Specifically, our initial model included the following predictors: group (an indicator variable coding for $\mathrm{NC}, \mathrm{AD}$, and $\mathrm{VaD})$, age, gender, number of ICA components removed during pre-processing, original sampling-rate, and number of clean epochs initially available; our dependent variable was a $[342 \times 6]$ matrix, which contained the six spectral parameters $(S, k, A, c$, $w$, and $b$ ) averaged over channels for the 342 subjects. Following stepwise reduction with an alpha value of 0.01 , the final model retained group $\left(T=76.23, p=7.1 \times 10^{-10}\right)$, age $(T=34.79$, $\left.p=1.7 \times 10^{-5}\right)$, gender $\left(T=23.89, p=1.0 \times 10^{-3}\right)$, and number of epochs $\left(T=38.7, p=3.8 \times 10^{-6}\right)$ as highly significant predictors. We then used these predictors in a second MANCOVA to model the spectral parameters at each channels, i.e., the dependent variable was a [342 $\times 132]$ matrix, representing 6 parameters at 22 channels, for all subjects. We confirmed that the four predictors (group, age, gender, and number of epochs) still accounted for significant variance in this larger model ( $p<0.005$ for all terms). Significance of the "group" factor at the channel level was determined from the $F$-statistics and corresponding $p$-values as shown in the Section "Results." $p$-Values were corrected for multiple comparisons using False Discovery Rate (FDR, $q=0.05$ ). For channels/parameters meeting significance, pair-wise contrasts were performed between groups (i.e., $\mathrm{NC}$ vs. $\mathrm{AD}, \mathrm{NC}$ vs. $\mathrm{VaD}$, and $\mathrm{AD}$ vs. $\mathrm{VaD}$ ). Pair-wise contrasts are denoted as significant at the (uncorrected) level of $p<0.05$, very significant at $p<0.01$, and highly significant at $p<0.001$, highlighted in Figures S1-S6 in Supplementary Material with “*”, " “**," and “***," respectively. For brevity, in presentation of our results, we determined the effect size for the group differences found at significant levels $p<0.05$ using Cohen's $d(48)$ and displayed on topographical maps as shown in Figure 6.

\section{RESULTS \\ GROUP SPECTRA}

Figure 3 displays the average spectra across all channels for the $\mathrm{NC}, \mathrm{AD}$, and $\mathrm{VaD}$ groups. Clear group differences were observed in the delta, theta, and alpha-frequency bands. These differences were quantified by comparing the six parameters $(S, k, A, c, w$, and 

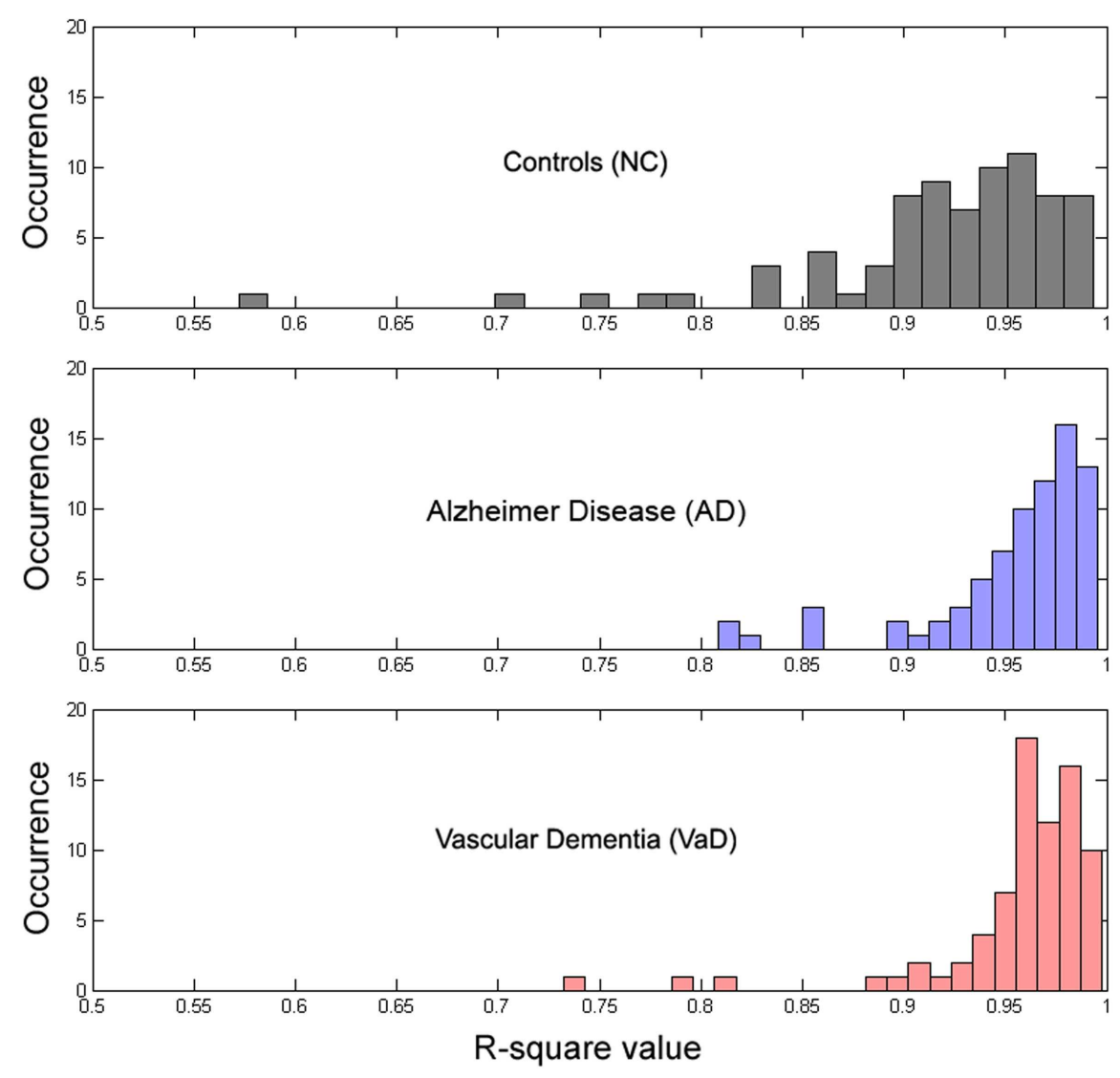

FIGURE 4 | Histogram of the distribution of $R^{2}$ values for each group.
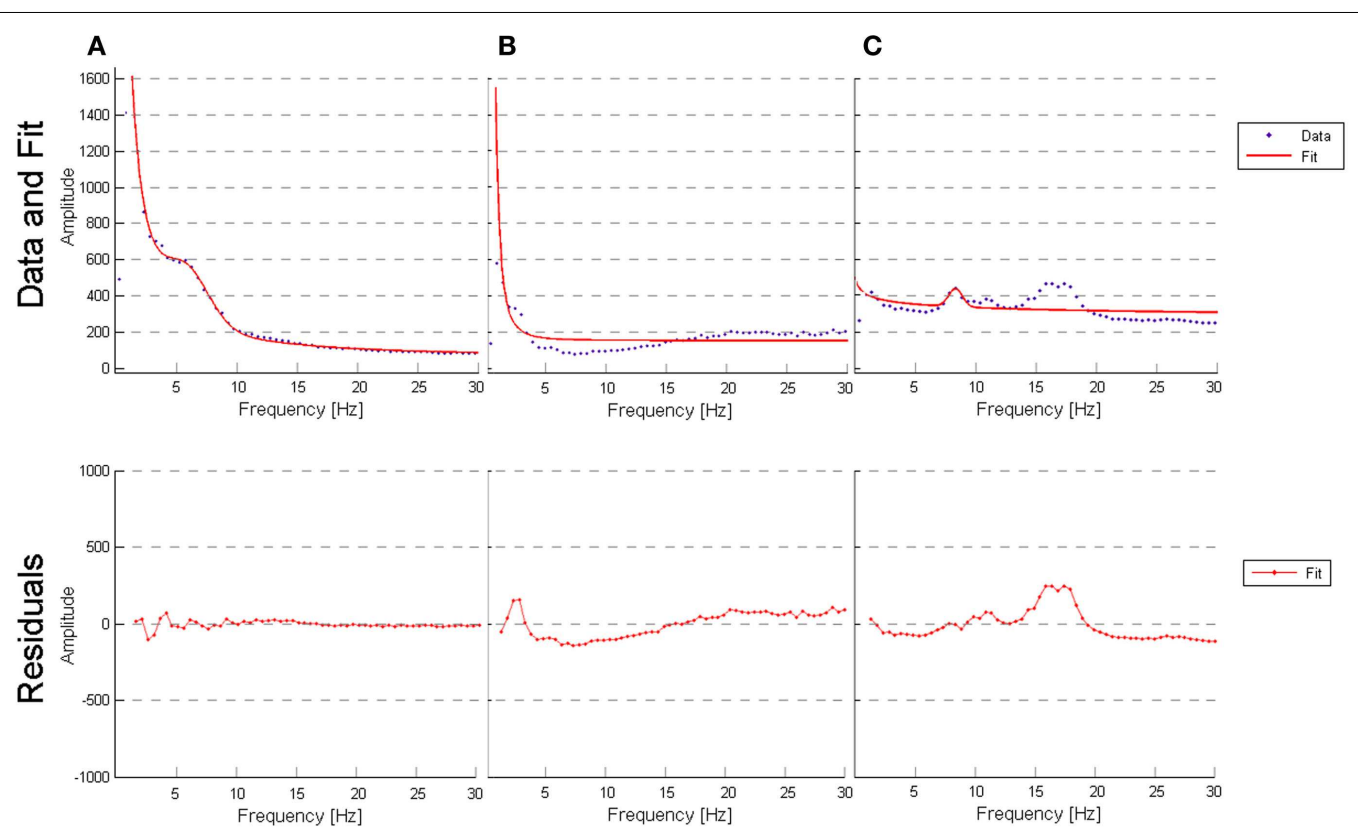

FIGURE 5 | (A) Outlier for best fit performed by the model (AD patient, channel T6, R-square value of 0.9955); (B) outlier for worst fit performed by the model (VaD patient, channel Fp2, R-square value of 0.7324); (C) outlier for worst fit performed by the model (subject from NC, channel O1, R-square value of 0.5724). 


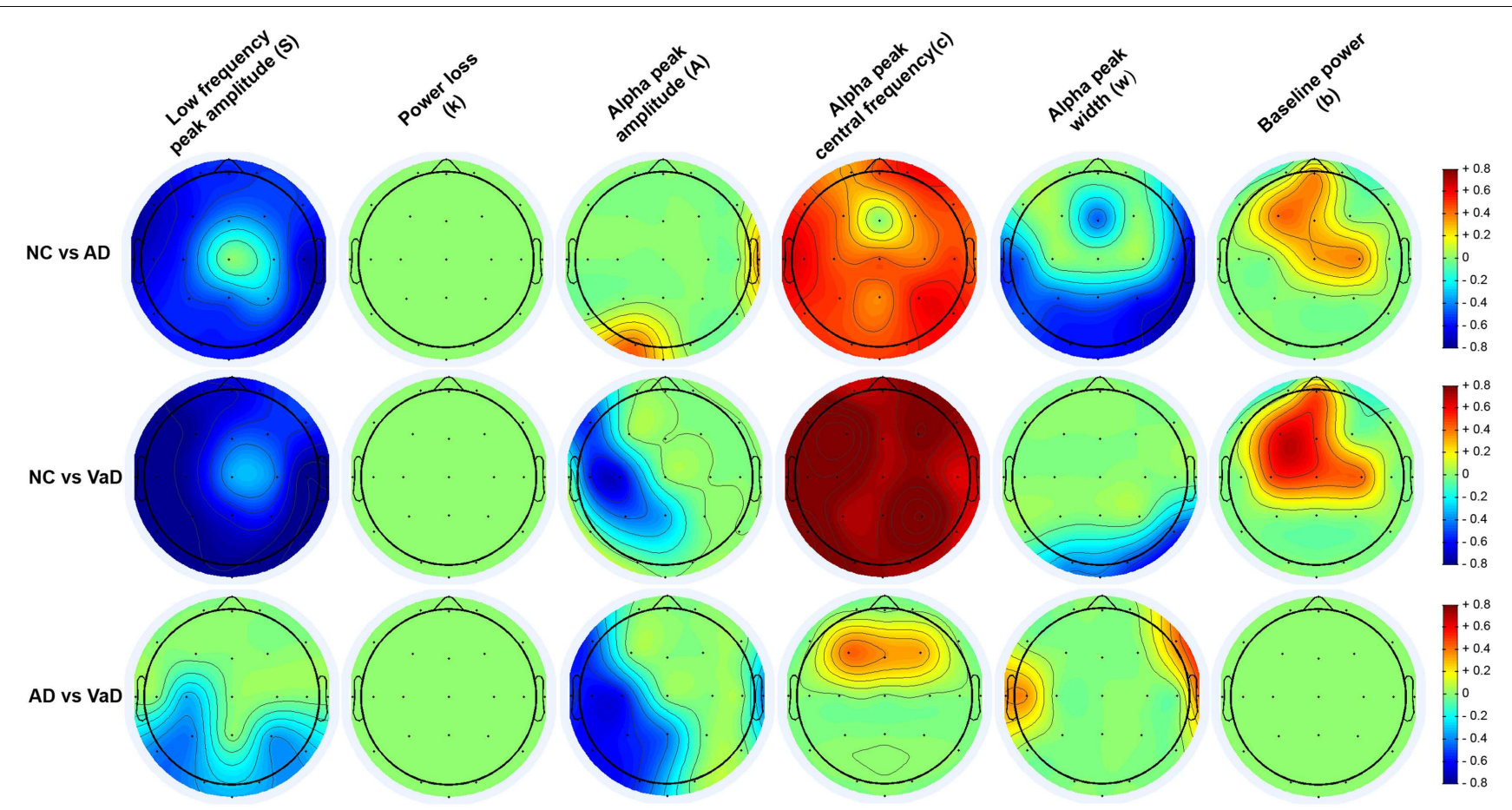

FIGURE 6 |Topography of the parameters effect sizes $(\boldsymbol{S}, \boldsymbol{k}, \boldsymbol{A}, \boldsymbol{c}, \boldsymbol{w}$, and $\boldsymbol{b}$ ) between groups (NC, AD, and VaD). Red or blue color gradients denote, respectively, a positive or negative effect when comparing the groups using Cohen's $d$ effect size scale $(|d|<0.2-$ small effect; $0.2<|d|<0.8-$ medium effect; $|d|>0.8$ - large effect).

b) used in the model, as described in the section "Materials and Methods."

\section{EVALUATION OF THE MODEL}

To determine whether our model fit the data well, we calculated the $R^{2}$ measure for each fit, reflecting the fraction of data variance captured by the model. Additionally, we computed the correlations between model parameters to check for dependence between parameters, which can indicate model redundancies or instabilities in the fitting procedure. Histograms of the $R^{2}$ values are displayed in Figure 4, and show excellent model fits in nearly all cases. The median goodness-of-fit was 0.96 and the first and third quartiles were 0.92 and 0.98 , respectively. We performed a Kruskal-Wallis one-way ANOVA test and no group differences were found in the $R^{2}$ values between the groups $\left(p<3.5 \mathrm{e}^{-8}\right)$, meaning that the model performed equally well fitting the datasets from each group. Examples of best and worst fits (as determined from the $R^{2}$ values) are shown in Figure 5. Only relatively weak correlations $(|r| \leq 0.4)$ between the parameters were found, with the exception of a high correlation between parameters $k$ and $b(r=0.74)$. This correlation was equally present within each group: $r=0.81$ for $\mathrm{NC}$, $r=0.64$ for $\mathrm{AD}$, and $r=0.79$ for $\mathrm{VaD}$.

\section{GROUP DIFFERENCES}

Group differences in spectral parameters were determined from the MANCOVA applied separately at each channel for all six parameters. For brevity, we report only findings that passed the FDR correction for significance (see Materials and Methods).
Figure 6 represents the topographical differences on a scale of Cohen's $d$ effect size, where $|d|<0.2$ is considered a small effect; $0.2<|d|<0.8$ medium effect; $|d|>0.8$ - large effect. For full details, readers are referred to Figures S1-S6 in Supplementary Material, which display the average parameter values, $F$-statistics, and $p$-values for each channel and group comparisons.

\section{[S] (low frequency power)}

As shown in Figure 6, both $\mathrm{AD}$ and particularly $\mathrm{VaD}$ patients had larger $S$ compared to NC. This difference was highly significant for most channels $\left(F>7, p<1.52 \times 10^{-3}\right)$. The VaD group also showed significantly higher $S$ than $\mathrm{AD}$ patients for parietal, temporal, and occipital channels $\left(F>17, p<1.51 \times 10^{-7}\right)$.

\section{[k] (1/f decay from lower to higher frequencies)}

No significant differences were found between $\mathrm{AD}, \mathrm{VaD}$, and $\mathrm{NC}$ group at group level.

\section{[A] (alpha power)}

Significant differences were observed in the $\mathrm{VaD}$ group compared with $\mathrm{NC}$, with $\mathrm{VaD}$ showing larger $A$ values over the left hemisphere and central channels $\left(F>4, p<2.36 \times 10^{-2}\right)$. VaD also showed significantly greater $A$ values compared with $\mathrm{AD}$ in nearly the same regions. AD, however, had significantly lower A than NC at occipital-left and lateral-right channels.

\section{[c] (alpha frequency)}

Vascular dementia patients had significantly lower $c$ values compared with NC for all channels $\left(F>8, p<8.43 \times 10^{-4}\right)$. The same 
trend was observed for AD compared with the NC group, where $\mathrm{AD}$ patients had lower $c$ values at all channels. At central and frontal channels, $\mathrm{VaD}$ showed lower $c$ compared with $\mathrm{AD}$. Mean $c$ values at occipital channels, were 9.4, 8.4, and $8.1 \mathrm{~Hz}$ for $\mathrm{NC}, \mathrm{AD}$, and $\mathrm{VaD}$ patients, respectively.

\section{[w] (alpha dispersion)}

Higher $w$ was found in AD compared with NC at central, occipital, and parietal areas $\left(F>4, p<2.53 \times 10^{-2}\right)$, while $\mathrm{VaD}$ had higher $w$ at occipital and right-temporal channels compared with NC $\left(F>7, p<1.46 \times 10^{-3}\right)$ The AD group had significantly higher $w$ than VaD mainly at lateral electrodes $\left(F>5, p<1.65 \times 10^{-2}\right)$

\section{[b] (baseline of the entire frequency spectrum)}

For the parameter $b$, significant differences were found between $\mathrm{NC}$ and both $\mathrm{AD}$ and $\mathrm{VaD}$ groups. Both $\mathrm{AD}$ and $\mathrm{VaD}$ groups displayed significantly lower $b$ than NC at central and frontal channels $\left(F>4, p<2.5 \times 10^{-2}\right)$.

\section{DISCUSSION}

This study explored electrophysiological differences that might be used as markers to automatically distinguish between groups. In order to assess those differences, we implemented quantitative analysis in clinical EEGs of $\mathrm{NC}, \mathrm{AD}$, and $\mathrm{VaD}$ and applied a model that extracted six EEG parameters to describe the average spectrum from each channel. Preliminary results (Figure 3) indicated marked differences between the three groups. Our approach uses standard models with relatively simple implementation and low computational costs. The EEG features extracted by our model are related to key relevant EEG markers for clinical assessment. We tested our model in $\mathrm{AD}$ and $\mathrm{VaD}$ group differentiation using the extracted features. Significant differences were found for several of the parameters with topographical specificity between AD and $\mathrm{VaD}$ in relation to healthy subjects. Our results inspire the use of such model as a standard approach for the extraction of EEG features and possible future use of such features as biomarkers in group differentiation.

\section{SPECTRUM MODEL}

The curve-fitting approach allowed us to describe the frequency spectrum using only six parameters, which are related with known electrophysiological markers. In general, we found that the model is a good fit to the data most of the time and that performs equally well regardless of the type of participant (see "Evaluation of the model" in "Results" section). We observed that a poor fit occurred in two situations: (1) subjects/channels where the spectrum curves contained a clear and distinguished theta peak that was distinct from alpha; (2) subjects/channels where low beta frequency amplitude was pronounced (as seen in Figure 5). Additionally, a correlation between the six parameters verified that parameters $k$ and $b$ are highly correlated $(r=0.75)$. This correlation points to a weakness in the model, since it appears that parameters $k$ and $b$ may work against each other.

\section{GROUP DIFFERENCES}

\section{Patients vs. controls}

Our results revealed significant increase of $S$ and a decrease of $b$ comparing the $\mathrm{AD}$ group with $\mathrm{NC}$, in line with previous literature (49). Parameter $c$ was $9.4 \mathrm{~Hz}$ in $\mathrm{NC}$ compared to $8.5 \mathrm{~Hz}$ in
AD. Similar alpha slowing from 10.2 to $8.1 \mathrm{~Hz}$ has been reported previously (50). Our study also indicates that AD patients have lower $A$ than NC. Slowing of alpha frequency and decreased alpha power have been reported in several studies, when comparing AD and also MCI patients with healthy controls $(36,50-55)$. This is thought to be associated with general cognitive decline (10-13). $\mathrm{AD}$ patients had higher $w$ than NC, in agreement with the findings from (47). VaD also had increased $S$, lower $b$, and increased $A$ compared with NC. The decrease of the $c$ was 9.4 to $8.1 \mathrm{~Hz}$ when compared with NC. (50) reported the corresponding values as $10.2-8.3 \mathrm{~Hz}$. In contrast to the $\mathrm{AD}$ group, $\mathrm{VaD}$ had higher $A$ in occipital regions than $\mathrm{NC}$, in accordance with previous studies (45, $56,57)$. It is well established that the source of the alpha rhythm is clearly predominant at occipital cortical regions (58). Other studies suggest that for $\mathrm{AD}$ and $\mathrm{VaD}$ patients, the increase of the alpha power is positively correlated with the glucose metabolism in the occipital lobe whereas the increase at lower frequencies of the EEG is negatively correlated with metabolism (59).

\section{VaD vs. AD}

Our results show significant differences for the $S$ parameter between $\mathrm{VaD}$ and $\mathrm{AD}$, in particular, an increase of $S$ for the $\mathrm{VaD}$ group for occipital, temporal, and parietal channels compared to the AD group, see Figures $\mathbf{3}$ and $\mathbf{6}$. In addition, VaD had significantly higher $A$ when compared with $\mathrm{AD}$ at occipital, temporal, lateral, and frontal electrodes. Interestingly, we observe a more pronounced decrease in $c$ for the $\mathrm{VaD}$ patients. Only very few studies have performed spectral comparisons between these two groups. Nevertheless, the slowing of alpha rhythm and the increase of power in the delta and theta band were more pronounced in $\mathrm{VaD}$ than in $\mathrm{AD}$ patients, as reported in the literature $(50,60)$. The general increase of $S$ along with decrease in $b$ and further slowing of $c$ have been reported in other studies and was suggested the use of such markers for accessing cognitive function (10-13) in MCI and $\mathrm{AD}$ studies.

Parameter $w$ differentiates patients with $\mathrm{AD}$ both from $\mathrm{VaD}$ and from NC at several channels. This parameter is similar to the dominant frequency variability (47), which was found to differentiate between AD, Lewy body, and Parkinson's disease. It is known that occipital frequency differences persist both in $\mathrm{AD}$ and $\mathrm{VaD}$ patients (45). Furthermore, at early stages of the disease, the neuropsychological and behavioral tests provide subtle differences leading to miss diagnoses of $\mathrm{AD}$ in $\mathrm{VaD}$ patients or vice-versa $(6,7)$. Therefore, we believe particularly this parameter $w$ to be an interesting biomarker for group differentiation.

\section{LIMITATIONS}

As we discussed previously, the EEG datasets that we included in our study were collected during normal clinical routine from the hospital and we relied on the latest existing clinical working ICD10 diagnosis determined by neurologists, (geronto-)psychiatrists, and neuropsychologists' staff. Despite the stringent criteria used to select these patients, there is still an uncertainty on diagnosing VaD or $\mathrm{AD}$, which can only be confirmed by a post-mortem test. More detailed reports of patient performance on various tests would require full access to patient data, which was not feasible here. Also, we have not correlated EEG findings with clinical scores such 
as MMSE, such that we cannot speculate about the relationships between severity of dementia symptoms and EEG abnormalities. Therefore, the group differences described in this study are biased to the uncertainty of diagnoses of our sample and should not per se be fully generalized.

\section{CONCLUSION}

Since this study included a large sample of unmedicated, age, and gender matched of $\mathrm{AD}, \mathrm{VaD}$, and $\mathrm{NC}$ with clinical EEGs, it was possible to compare the spectral differences between these groups simultaneously. The model used in this study extracted relevant EEG features such as low and high frequency amplitudes, decay of amplitude from low to high frequencies, alpha frequency, alpha power, and dispersion of alpha frequency. The implementation of this model is suitable for analyzing EEG, and has relatively low time and processing costs by reducing the complexity of the broad spectrum curve. Our findings revealed significant differences in several of the features extracted by the model, namely lower alpha power at lateral-occipital regions for $\mathrm{AD}$ patients when compared with NC in contrast to $\mathrm{VaD}$ patients who had higher power compared with $\mathrm{NC}$ for the same regions. Both $\mathrm{AD}$ and $\mathrm{VaD}$ patients had higher delta power than NC and, for posterior channels. VaD patients had even higher delta power than $\mathrm{AD}$ patients. The dispersion of alpha, $w$, was a novel feature extracted by our fit-curve model and established quantifiable differences between $\mathrm{AD}$ and $\mathrm{VaD}$. Based on this study, we believe that the proposed model is useful for extraction and quantification of differences between these groups of patients, and may have the potential to be used as a general tool in quantitative EEG analysis.

\section{SUPPLEMENTARY MATERIAL}

The Supplementary Material for this article can be found online at http://www.frontiersin.org/Journal/10.3389/fneur.2015.00025/ abstract

\section{REFERENCES}

1. Jeong J. EEG dynamics in patients with Alzheimer's disease. Clin Neurophysiol (2004) 115(7):1490-505. doi:10.1016/j.clinph.2004.01.001

2. Mattson MP. Pathways towards and away from Alzheimer's disease. Nature (2004) 430(7000):631-9. doi:10.1038/nature02621

3. Meek PD, McKeithan K, Schumock GT. Economic considerations in Alzheimer's disease. Pharmacotherapy (1998) 18(2 Pt 2):68-73.

4. Roman GC. Vascular dementia revisited: diagnosis, pathogenesis, treatment, and prevention. Med Clin North Am (2002) 86(3):477-99. doi:10.1016/S00257125(02)00008-1

5. Dubois MF, Hebert R. The incidence of vascular dementia in Canada: a comparison with Europe and East Asia. Neuroepidemiology (2001) 20(3):179-87. doi:10.1159/000054785

6. Gearing M, Mirra SS, Hedreen JC, Sumi SM, Hansen LA, Heyman A. The consortium to establish a registry for Alzheimer's disease (CERAD). Part X. Neuropathology confirmation of the clinical diagnosis of Alzheimer's disease. Neurology (1995) 45(3 Pt 1):461-6. doi:10.1212/WNL.45.3.461

7. Massoud F, Devi G, Stern Y, Lawton A, Goldman JE, Liu Y, et al. A clinicopathological comparison of community-based and clinic-based cohorts of patients with dementia. Arch Neurol (1999) 56(11):1368-73. doi:10.1001/archneur.56. 11.1368

8. Fiandaca MS, Mapstone ME, Cheema AK, Federoff HJ. The critical need for defining preclinical biomarkers in Alzheimer's disease. Alzheimers Dement (2014) 10(3 Suppl):S196-212. doi:10.1016/j.jalz.2014.04.015

9. Weiner MW, Veitch DP, Aisen PS, Beckett LA, Cairns NJ, Green RC, et al. The Alzheimer's disease neuroimaging initiative: a review of papers published since its inception. Alzheimers Dement (2013) 9(5):e111-94. doi:10.1016/j.jalz.2013. 05.1769

10. Dierks T, Frölich L, Ihl R, Maurer K. Correlation between cognitive brain function and electrical brain activity in dementia of Alzheimer type. J Neural Transm Gen Sect (1995) 99(1-3):55-62. doi:10.1007/BF01271469

11. Kwak YT. Quantitative EEG findings in different stages of Alzheimer's disease. JClin Neurophysiol (2006) 23(5):456-61. doi:10.1097/01.wnp.0000223453. 47663.63

12. Anghinah R, Kanda PA, Lopes HF, Basile LF, Machado S, Ribeiro P, et al. Alzheimer's disease qEEG: spectral analysis versus coherence. Which is the best measurement? Arq Neuropsiquiatr (2011) 69(6):871-4. doi:10.1590/S0004282X2011000700004

13. Babiloni C, Infarinato F, Marzano N, Iacoboni M, Dassù F, Soricelli A, et al. Intra-hemispheric functional coupling of alpha rhythms is related to golfer's performance: a coherence EEG study. Int J Psychophysiol (2011) 82(3):260-8. doi:10.1016/j.ijpsycho.2011.09.008

14. Blennow K, Hampel H. CSF markers for incipient Alzheimer's disease. Lancet Neurol (2003) 2(10):605-13. doi:10.1016/S1474-4422(03)00530-1

15. Cichocki A, Shishkin SL, Musha T, Leonowicz Z, Asada T, Kurachi T. EEG filtering based on blind source separation (BSS) for early detection of Alzheimer's disease. Clin Neurophysiol (2005) 116(3):729-37. doi:10.1016/j.clinph.2004.09.017

16. DeKosky ST, Marek K. Looking backward to move forward: early detection of neurodegenerative disorders. Science (2003) 302(5646):830-4. doi:10.1126/ science. 1090349

17. Wagner AD. Early detection of Alzheimer's disease: an fMRI marker for people at risk? Nat Neurosci (2000) 3(10):973-4. doi:10.1038/79904

18. Rapoport SI. Functional brain imaging to identify affected subjects genetically at risk for Alzheimer's disease. Proc Natl Acad Sci U S A (2000) 97(11):5696-8. doi:10.1073/pnas.120178897

19. Schomer DL, Silva FHLD. Electroencephalography. In: Niedermeyer E, Schomer DL, Silva FHLD, editors. Niedermeyer's Electroencephalography. Basic Principles, Clinical Applications, and Related Fields. Philadelphia: Wolters Kluwer/Lippincott Williams \& Wilkins Health (2011). p. 911-22.

20. Bauer G, Bauer R. Electroencephalography. In: Niedermeyer E, Schomer DL, Silva FHLD, editors. Niedermeyer's Electroencephalography. Chapter 43: EEG, Drug Effect, and Central Nervous System Poisoning. Philadelphia: Wolters Kluwer/Lippincott Williams \& Wilkins Health (2011). p. 911-22.

21. Jelic V, Johansson SE, Almkvist O, Shigeta M, Julin P, Nordberg A, et al. Quantitative electroencephalography in mild cognitive impairment: longitudinal changes and possible prediction of Alzheimer's disease. Neurobiol Aging (2000) 21(4):533-40. doi:10.1016/S0197-4580(00)00153-6

22. Prichep LS. Quantitative EEG and electromagnetic brain imaging in aging and in the evolution of dementia. Ann N Y Acad Sci (2007) 1097:156-67. doi:10.1196/annals.1379.008

23. Prichep LS, John ER, Ferris SH, Rausch L, Fang Z, Cancro R, et al. Prediction of longitudinal cognitive decline in normal elderly with subjective complaints using electrophysiological imaging. Neurobiol Aging (2006) 27(3):471-81. doi:10.1016/j.neurobiolaging.2005.07.021

24. Rossini PM, Del Percio C, Pasqualetti P, Cassetta E, Binetti G, Dal Forno G, et al. Conversion from mild cognitive impairment to Alzheimer's disease is predicted by sources and coherence of brain electroencephalography rhythms. Neuroscience (2006) 143(3):793-803. doi:10.1016/j.neuroscience.2006.08.049

25. Rossini PM, Rossi S, Babiloni C, Polich J. Clinical neurophysiology of aging brain: from normal aging to neurodegeneration. Prog Neurobiol (2007) 83(6):375-400. doi:10.1016/j.pneurobio.2007.07.010

26. Klassen BT, Hentz JG, Shill HA, Driver-Dunckley E, Evidente VG, Sabbagh MN, et al. Quantitative EEG as a predictive biomarker for Parkinson disease dementia. Neurology (2011) 77(2):118-24. doi:10.1212/WNL.0b013e318224af8d

27. Muresanu DF, Alvarez XA, Moessler H, Novak PH, Stan A, Buzoianu A, et al. Persistence of the effects of Cerebrolysin on cognition and qEEG slowing in vascular dementia patients: results of a 3-month extension study. J Neurol Sci (2010) 299(1-2):179-83. doi:10.1016/j.jns.2010.08.040

28. Gawel M, Zalewska E, Szmidt-Sałkowska E, Kowalski J. The value of quantitative EEG in differential diagnosis of Alzheimer's disease and subcortical vascular dementia. J Neurol Sci (2009) 283(1-2):127-33. doi:10.1016/j.jns.2009.02.332

29. Schreiter Gasser U, Rousson V, Hentschel F, Sattel H, Gasser T. Alzheimer disease versus mixed dementias: an EEG perspective. Clin Neurophysiol (2008) 119(10):2255-9. doi:10.1016/j.clinph.2008.07.216 
30. Dierks T, Perisic I, Frölich L, Ihl R, Maurer K. Topography of the quantitative electroencephalogram in dementia of the Alzheimer type: relation to severity of dementia. Psychiatry Res (1991) 40(3):181-94. doi:10.1016/0925-4927(91) 90009-F

31. Prichep LS, John ER, Ferris SH, Reisberg B, Almas M, Alper K, et al. Quantitative EEG correlates of cognitive deterioration in the elderly. Neurobiol Aging (1994) 15(1):85-90. doi:10.1016/0197-4580(94)90147-3

32. Pucci E, Cacchiò G, Angeloni R, Belardinelli N, Nolfe G, Signorino M, et al. EEG spectral analysis in Alzheimer's disease and different degenerative dementias. Arch Gerontol Geriatr (1998) 26(3):283-97. doi:10.1016/S0167-4943(98) 00012-0

33. Huang C, Wahlund L, Dierks T, Julin P, Winblad B, Jelic V. Discrimination of Alzheimer's disease and mild cognitive impairment by equivalent EEG sources: a cross-sectional and longitudinal study. Clin Neurophysiol (2000) 111(11):1961-7. doi:10.1016/S1388-2457(00)00454-5

34. Fraga FJ, Falk TH, Kanda PA, Anghinah R. Characterizing Alzheimer's disease severity via resting-awake EEG amplitude modulation analysis. PLoS One (2013) 8(8):e72240. doi:10.1371/journal.pone.0072240

35. Lehmann C, Koenig T, Jelic V, Prichep L, John RE, Wahlund LO, et al. Application and comparison of classification algorithms for recognition of Alzheimer's disease in electrical brain activity (EEG). J Neurosci Methods (2007) 161(2):342-50. doi:10.1016/j.jneumeth.2006.10.023

36. Dauwels J, Vialatte F, Cichocki A. Diagnosis of Alzheimer's disease from EEG signals: where are we standing? Curr Alzheimer Res (2010) 7(6):487-505. doi: $10.2174 / 156720510792231720$

37. Aurlien H, Gjerde IO, Gilhus NE, Hovstad OG, Karlsen B, Skeidsvoll H. A new way of building a database of EEG findings. Clin Neurophysiol (1999) 110(5):986-95. doi:10.1016/S1388-2457(99)00037-1

38. Delorme A, Makeig S. EEGLAB: an open source toolbox for analysis of singletrial EEG dynamics including independent component analysis. JNeurosci Methods (2004) 134(1):9-21. doi:10.1016/j.jneumeth.2003.10.009

39. Welch BL. The significance of the difference between two means when the population variances are unequal. Biometrika (1938) 29:350-62. doi:10.1093/biomet/ 29.3-4.350

40. Lehmann D, Skrandies W. Reference-free identification of components of checkerboard-evoked multichannel potential fields. Electroencephalogr Clin Neurophysiol (1980) 48(6):609-21. doi:10.1016/0013-4694(80)90419-8

41. Penttilä M, Partanen JV, Soininen H, Riekkinen PJ. Quantitative analysis of occipital EEG in different stages of Alzheimer's disease. Electroencephalogr Clin Neurophysiol (1985) 60(1):1-6. doi:10.1016/0013-4694(85)90942-3

42. Miyauchi T, Hagimoto H, Ishii M, Endo S, Tanaka K, Kajiwara S, et al. Quantitative EEG in patients with presenile and senile dementia of the Alzheimer type. Acta Neurol Scand (1994) 89(1):56-64. doi:10.1111/j.1600-0404.1994.tb01633.x

43. Lipping T, Anier A, Ratsep I, Kleemann P, Toome V, Jantti V. Tracking rhythm in long-term EEG recordings using empirical mode calculation. Conf Proc IEEE Eng Med Biol Soc (2008) 2008:3604-7. doi:10.1109/IEMBS.2008.4649986

44. Del Percio C, Iacoboni M, Lizio R, Marzano N, Infarinato F, Vecchio F, et al. Functional coupling of parietal alpha rhythms is enhanced in athletes before visuomotor performance: a coherence electroencephalographic study. Neuroscience (2011) 175:198-211. doi:10.1016/j.neuroscience.2010.11.031

45. Tsuno N, Shigeta M, Hyoki K, Faber PL, Lehmann D. Fluctuations of source locations of EEG activity during transition from alertness to sleep in Alzheimer's disease and vascular dementia. Neuropsychobiology (2004) 50(3):267-72. doi: $10.1159 / 000079982$

46. Moretti DV, Zanetti O, Binetti G, Frisoni GB. Quantitative EEG markers in mild cognitive impairment: degenerative versus vascular brain impairment. Int J Alzheimers Dis (2012) 2012:917537. doi:10.1155/2012/917537

47. Bonanni L, Thomas A, Tiraboschi P, Perfetti B, Varanese S, Onofrj M. EEG comparisons in early Alzheimer's disease, dementia with Lewy bodies and Parkinson's disease with dementia patients with a 2-year follow-up. Brain (2008) 131 $(\mathrm{Pt}$ 3):690-705. doi:10.1093/brain/awm322
48. Cohen J. The Statistical Power Analysis for the Behavioural Sciences. 2nd ed. Hillsdale, NJ: Lawrence Erlbaum Associates (1988).

49. Besthorn C, Zerfass R, Geiger-Kabisch C, Sattel H, Daniel S, Schreiter-Gasser U, et al. Discrimination of Alzheimer's disease and normal aging by EEG data. Electroencephalogr Clin Neurophysiol (1997) 103(2):241-8. doi:10.1016/S00134694(97)96562-7

50. Signorino M, Pucci E, Brizioli E, Cacchio G, Nolfe G, Angeleri F. EEG power spectrum typical of vascular dementia in a subgroup of Alzheimer patients. Arch Gerontol Geriatr (1996) 23(2):139-51. doi:10.1016/0167-4943(96)00714-5

51. Babiloni C, Lizio R, Del Percio C, Marzano N, Soricelli A, Salvatore E, et al. Cortical sources of resting state EEG rhythms are sensitive to the progression of early stage Alzheimer's disease. J Alzheimers Dis (2013) 34(4):1015-35. doi:10.3233/JAD- 121750

52. Schreiter-Gasser U, Gasser T, Ziegler P. Quantitative EEG analysis in early onset Alzheimer's disease: a controlled study. Electroencephalogr Clin Neurophysiol (1993) 86(1):15-22. doi:10.1016/0013-4694(93)90063-2

53. Coben LA, Danziger W, Storandt M. A longitudinal EEG study of mild senile dementia of Alzheimer type: changes at 1 year and at 2.5 years. Electroencephalogr Clin Neurophysiol (1985) 61(2):101-12. doi:10.1016/0013-4694(85) 91048-X

54. Roh JH, Park MH, Ko D, Park KW, Lee DH, Han C, et al. Region and frequency specific changes of spectral power in Alzheimer's disease and mild cognitive impairment. Clin Neurophysiol (2011) 122(11):2169-76. doi:10.1016/j.clinph. 2011.03.023

55. Babiloni C, Lizio R, Vecchio F, Frisoni GB, Pievani M, Geroldi C, et al. Reactivity of cortical alpha rhythms to eye opening in mild cognitive impairment and Alzheimer's disease: an EEG study. J Alzheimers Dis (2010) 22(4):1047-64. doi:10.3233/JAD-2010-100798

56. Lou W, Xu J, Sheng H, Zhao S. Multichannel linear descriptors analysis for event-related EEG of vascular dementia patients during visual detection task. Clin Neurophysiol (2011) 122(11):2151-6. doi:10.1016/j.clinph.2011.03.021

57. van Straaten EC, de Haan W, de Waal H, Scheltens P, van der Flier WM, Barkhof F, et al. Disturbed oscillatory brain dynamics in subcortical ischemic vascular dementia. BMC Neurosci (2012) 13:85. doi:10.1186/1471-2202-13-85

58. da Silva FH, van Lierop TH, Schrijer CF, van Leeuwen WS. Organization of thalamic and cortical alpha rhythms: spectra and coherences. Electroencephalogr Clin Neurophysiol (1973) 35(6):627-39. doi:10.1016/0013-4694(73)90217-4

59. Szelies B, Mielke R, Kessler J, Heiss WD. EEG power changes are related to regional cerebral glucose metabolism in vascular dementia. Clin Neurophysiol (1999) 110(4):615-20. doi:10.1016/S1388-2457(98)00052-2

60. Signorino M, Pucci E, Belardinelli N, Nolfe G, Angeleri F. EEG spectral analysis in vascular and Alzheimer dementia. Electroencephalogr Clin Neurophysiol (1995) 94(5):313-25. doi:10.1016/0013-4694(94)00290-2

Conflict of Interest Statement: The authors declare that the research was conducted in the absence of any commercial or financial relationships that could be construed as a potential conflict of interest.

Received: 06 July 2014; accepted: 29 January 2015; published online: 13 February 2015. Citation: Neto E, Allen EA, Aurlien H, Nordby H and Eichele T (2015) EEG spectral features discriminate between Alzheimer's and vascular dementia. Front. Neurol. 6:25. doi: 10.3389/fneur.2015.00025

This article was submitted to Brain Imaging Methods, a section of the journal Frontiers in Neurology.

Copyright (c) 2015 Neto, Allen, Aurlien, Nordby and Eichele. This is an open-access article distributed under the terms of the Creative Commons Attribution License (CC $B Y)$. The use, distribution or reproduction in other forums is permitted, provided the original author(s) or licensor are credited and that the original publication in this journal is cited, in accordance with accepted academic practice. No use, distribution or reproduction is permitted which does not comply with these terms. 